\title{
CONCERNING LIMITING SETS IN ABSTRACT SPACES*
}

BY

\section{R. G. LUBBEN}

By a limit point of a point set $M$ we mean a point which is the limit of an infinite sequence of distinct points belonging to $M$. This notion may be generalized by regarding a point set as being the limit, in some sense, of a collection of point sets. We shall give a precise formulation of such a generalzation: A point set $K$ is said to be the limiting set of a collection $G$ of point sets provided that it is the set of all points $P$ such that there exists a sequence of points $P_{1}, P_{2}, P_{3}, \cdots$, and a sequence $g_{1}, g_{2}, g_{3}, \cdots$, of distinct elements of $G$ such that $P$ is the limit of the sequence $P_{1}, P_{2}, P_{3}, \cdots$, and for every $n, P_{n}$ belongs to $g_{n} . \ddagger$ Theorems concerning such limiting sets are frequently

* Presented to the Society, September 10,1925, and September 6, 1928; received by the editors in June, 1926, and January, 1928. The author proved Theorems 1,16 , and 17 while holding a National Research Fellowship.

$\dagger$ The theorems in this paper will be stated for a space $L$ Fréchet or for a specialization of such a space. A space $L$ is a class of elements, which we shall call points, such that (1) if $P$ is an element of $L$ and $P_{1}, P_{2}, P_{3}, \cdots$ is a countable sequence of elements belonging to $L$, then the statement $P$ is the limit of the sequence $P_{1}, P_{2}, P_{3}, \cdots$ has a definite meaning and the question whether this statement is true or false has a determinate answer as soon as the element $P$ and the sequence in question are themselves determined; (2) if the element $P$ is the limit of the sequence $P_{1}, P_{2}, P_{3}, \cdots$, and $n_{1}$, $n_{2}, n_{3}, \cdots$ is a sequence of positive integers such that $n_{1}<n_{2}<n_{3}<\cdots$ then $P$ is the limit of $P_{n(1)}, P_{n(2)}, P_{n(3)}, \cdots$ (where, for typographical reasons, $n(i)$ is used in subscripts in place of $\left.n_{i}\right) ;(3)$ if $P$ is an element of $L, P$ is the limit of the sequence $P, P, P, \ldots$ all of whose elements coincide with the point $P$. Cf. M. Fréchet, (I) Sur quelques points du calcul fonctionnel, Rendiconti del Circolo Matematico di Palermo, vol. 22 (1906), pp. 5-6. A space $D$ is a space $L$ in which with every pair of points $A$ and $B$ there is associated a number $d(A, B)$ such that $(1) d(A, B)=d(B, A) \geqq 0$, (2) $d(A, B)=0$ if and only if $A=B$, (3) if $A, B$, and $C$ are any three elements then $d(A, B)+d(B, C)$ $\geqq d(A, C)$, and (4) the limit of the sequence $P_{1}, P_{2}, P_{3}, \cdots$ is the point $P$ if and only if $d\left(P_{n}, P\right)$ approaches zero as $n$ approaches infinity. Cf. Fréchet, loc. cit., p. 30, and also (II), Les ensembles abstraits et le calcul fonctionnel, Rendiconti del Circolo Matematico di Palermo, vol. 30 (1910), p. 2, paragraph 3, and (III), Sur les ensembles abstraits, Annales Scientifiques de l'Ecole Normale Superieure, (3), vol. 38 (1921), p. 350, lines 1-10. The symbol $d(A, B)$ is read "the distance from $A$ to $B . "$

† C. R. L. Moore, Report on continuous curves from the view-point of analysis situs, Bulletin of the American Mathematical Society, vol. 29 (1923), p. 297. Concerning variations of this definition (mostly for less general spaces), and also of definitions, to be given later, of other types of limiting sets, see the following papers: L. Zoretti, (I), Sur les fonctions analytiques uniformes qui posseddent un ensemble parfait discontinu de points singuliers, Journal de Mathématiques Pures et Appliquees, (6), vol. 1 (1905), pp. 8-9, and (II), Un théorème de la theorie des ensembles, Bulletin de la Société Mathematique de France, vol. 37 (1909), pp. 116-119; A. Schoenflies, Bemerkung zu meinem zweilen Beitrag zur Theorie der Punktmengen, Mathematische Annalen, vol. 65 (1908), pp. 431-432; S. Janis- 
applicable in point set theory and in abstract topology; as an example we may mention theorems concerning conditions under which the limiting set is connected (cf. Theorems 11 and 12, and the discussion and references there given).

It is of interest to discover what properties hold for limiting sets in euclidean spaces, and to determine the general classes of abstract spaces in which such theorems hold. One of the purposes of the study of abstract spaces is the discovery of conditions, preferably necessary and sufficient, that a given theorem should hold in that space. As an example we may consider the result shown in Theorem 1 of this paper. In ordinary space the limiting set of every collection of point sets is closed. We show in Theorem 1 that in a space $L$ a necessary and sufficient condition that the limiting set of every infinite collection of point sets be closed is that the space under consideration be a space $S .^{*}$ Thus, the majority of the properties of a euclidean space have little bearing on the fact that in such a space the limiting set of a collection of point sets is closed.

Another example of such a study is the one we make of the distributive property. A space is said to have the distributive property $\dagger$ or to be distributive provided that if in that space $K$ is a closed point set and $G$ is a collection of point sets and each point of $K$ belongs to some sub-set of $K$ which is the limiting set of some sub-collection of $G$, then $K$ is itself the limiting set of a sub-collection $G_{1}$ of $G$. If it be specified in addition that $G_{1}$ be countable, the space is said to have the countably distributive property or to be countably distributive. We show in this paper that every distributive space $D$ is locally compact $\ddagger$ and further that in order that a space $D$ have the countably

zewski, Sur les continus irréductibles entre deux points, Journal de l'Ecole Polytechnique, (2), vol. 16 (1912), pp.93-94; F.Hausdorf, (I), Grundzüge der Mengenlehre, Leipzig, Veit, 1914, pp. 233-239 and 296-304, and (II), Mengenlehre, Berlin, de Gruyter, 1927, pp. 145-150; L. Vietoris, Stetige Mengen, Monatshefte für Mathematik und Physik, vol. 31 (1921), pp. 184-194; Zoretti-Rosenthal, Mengen die von einem Parameter abhangen, Encyklopaedie der Mathematischen Wissenschaften, Band II, 3, Heft 7 (II C 9a), \$15, pp. 938-941.

* The derived set of a point set is the set of all its limit points. A space $S$ is a space $L$ in which the derived set of every point set is closed. Cf. M. Fréchet, (IV), Relations entre les notions de limite et de distance, these Transactions, vol. 19 (1918), p. 55. If $M$ is a point set, $M^{\prime}$ denotes the derived set of $M$, and $\bar{M}=M+M^{\prime}$. $M \subset N$ means that $M$ is a sub-set of $N$.

$\dagger$ The term is due to R. L. Moore. The question whether a bounded euclidean space has this property was raised by W. L. Ayres during a discussion of the proof of a special case of my Theorem 13, and was settled in the affirmative by R. L. Moore.

¥ A point set is said to be compact provided that each of its infinite sub-sets has at least one limit point. (Cf. Fréchet I, pp. 6-7.) A space $D$ is said to be locally compact at a point $P$ or to be compact near $P$ provided that $P$ belongs to a compact open set. A point set is said to be an open set provided that its complement in the space under consideration is closed. A space is locally compact provided that it is locally compact at each of its points. A point set is separable provided that it 
distributive property it is necessary and sufficient that it be locally compact and separable.

Certain theorems concerning limiting sets have an analogy to covering theorems. The question concerning the possibility of replacing any collection of point sets in a space by a countable sub-collection having the same limiting set is analogous to the one whether the Lindelöf property* holds in the space. This question is, however, of less interest than the following one: Under what conditions is a point set the limiting set of a given collection of point sets? (See Theorems 9, 10, 12, 13, and 14.) The distributive property is of especial interest in this connection; in a space possessing this property we have a condition which may be described roughly as follows: If the property of being the limiting set of a sub-collection of $G$ is distributed everywhere in a closed point set $K$, then $K$ itself has this property. It was for the reason just indicated that the term "distributive property" suggested itself to Moore.

Return to the consideration of a hyperspace whose elements are point sets in a given space. Condition (2) mentioned in the definition of a space $L$ is not satisfied if we interpret "the limit of a sequence" as the limiting set of a sequence of point sets. $\dagger$ This objection does not apply to the sequential limiting set. The limiting set of an infinite collection of point sets is called the sequentialf limiting set of this collection if and only if every infinite subcollection of the given collection has the same limiting set; a collection of point sets is convergent if it has a non-vacuous sequential limiting set and is divergent if it has a vacuous sequential limiting set. $\$$ In Theorems 16 and 17 we show that the aggregate of all closed point sets in an abstract space may be regarded as a space $L$ provided that we introduce a suitable definition for "the limit of a sequence," which involves the notion of a sequential limiting set. In this connection Theorems 5, 6, and 7 are of interest; Theorem 6 shows that there exist spaces $L$ and even spaces $D$ in which no collection of point sets contains a convergent or a divergent sub-sequence.

Janiszewskill gives a definition of the limit set of a sequence of point sets;

contains a countable sub-set such that every point of the set either belongs to this sub-set or is a limit point of it. Cf. Fréchet III, p. 341.

* A collection of open sets is said to cover a point set $K$ provided that each point of $K$ belongs to some element of the given collection. A space is said to have the Lindelöf property provided that for every collection of open sets covering a point set in it there exists a countable sub-collection covering the point set. Lindelöf first showed that a euclidean space has this property; cf. his paper, Remarques sur un theorème fondamental de la theorie des ensembles, Acta Mathematica, vol. 29 (1905), p. 188.

† Cf. Janiszewski, loc. cit., pp. 94-95, example 1.

$\ddagger$ Cf. R. L. Moore, loc. cit., and Hausdorff I, loc. cit.

Cf. Hausdorf I, pp. 230 and 232.

|| Loc. cit., p. 94. 
we modify his definition as follows: The limit set of a collection of point sets is the set of all points $Q$ such that $Q$ belongs to the limiting set of every infinite sub-collection of the given collection. It follows from our definition that if $P$ is a point in the limiting set of a collection $G$ of point sets, then $P$ belongs to the limit set of some infinite sub-collection of $G$; we show in Theorem 6 that it is not true in general, even in a space $D$, that $P$ belongs to the sequential limiting set of a sub-collection of $G$.

Janiszewski* shows that the limit set may be a proper sub-set of the limiting set, and may even be vacuous. In fact, the sequential limiting set exists if and only if the limit set is the same as the limiting set; and, in case this is true, the three are identical. The limiting set of an infinite subcollection of a collection of point sets is a sub-set of the limiting set of the collection. It is not implied in our definitions that all the elements of a collection of point sets should be distinct; it is possible, for instance that each element should consist of a single point, and that all are the same point; in this case the limiting set of the collection would be the given point.

In concluding the introduction the author wishes to thank R. L. Moore, who indicated to one of his classes the desirability of a systematic study of limiting sets, and whose criticisms and suggestions have been of great value in the preparation of this paper.

THEOREM 1. In order that every collection of point sets in a spaceL should have a closed limiting set it is necessary and sufficient that the space be a space $S$.

If the condition is not sufficient, there must exist a space $S$ in which there exists a collection $G$ of point sets whose limiting set is not closed. Let $P$ be a point which does not belong to $K$, the limiting set of $G$, but is the sequential limit point $\dagger$ of a sequence $U=Q_{1}+Q_{2}+Q_{3}+\cdots$ of distinct points belonging to $K$. Let $F_{n}$ be the aggregate of all elements $g$ of $G$ such that, for some integer $m>n, g$ contains the point $Q_{m}$. It follows that $F_{n+1} \subset F_{n}$. Then for some sufficiently large value $N$ of $n, F_{N}$ will contain at most a finite number of elements; otherwise, as may easily be seen, $P$ would have to belong to the limiting set of $G$. Let $F=G-F_{N}, P_{i}=Q_{N+i}$, and $T=\sum P_{i}(i=1,2,3, \cdots)$.

It follows from the definition of $P_{i}$ and the fact that $P_{i}$ is a point of no element of $F$ that there exists for each positive integer $i$ a sequence

- Loc. cit.

$\dagger$ A point is the sequential limit point of a sequence of points $M$ provided that it is the limit of every infinite sub-sequence of $M$. Cf. R. L. Moore, On the foundations of plane analysis situs, these Transactions, vol. 17 (1916), p. 134. 
$T_{i}=\sum P_{i i}(j=1,2,3, \cdots)$ of distinct points such that (1) $P_{i}$ is the sequential limit point of $T_{i}$ and (2) for each integer $i, P_{i i}$ belongs to an element $g_{i i}$ of $F$ such that if $m \neq n$ then $g_{m i} \neq g_{n i}$.

Let $S_{1}=Q_{11}=P_{11}$ and let $h_{11}=G_{1}$ be a definite element of $F$ containing $P_{11}$. For $k>1$ let $G_{k}=\sum h_{k i}(i=1,2,3, \cdots, k)$ be a sequence of elements of $F$ and $S_{k}=\sum Q_{k i}(i=1,2,3, \cdots, k)$ be a sequence of points selected as follows: $h_{k i}$ is an element of $\sum g_{i i}(j=1,2,3, \cdots, \infty)$ with $j=n_{k i}$, where $n_{k i}$ is the smallest positive integer $x$ such that $g_{x i}$ is not one of the finite number of elements of $F$ belonging to $G_{1}, G_{2}, \cdots, G_{k-1} ; Q_{k i}$ is the point $P_{n(k, i), i .}{ }^{*}$ Let $W=\sum S_{i}(i=1,2,3, \cdots, \infty)$. For every integer $i, W$ contains infinitely many points in common with $T_{i}$, and hence $T$ is a sub-set of the derived set of $W$. Since $W$ is a sub-set of a space $S$, its derived set is closed. It follows that there exists a set $Q_{k(1), i(1)}, Q_{k(2), i(2)}, Q_{k(3), i(3)}, \cdots$, of distinct points of $W$ having $P$ as a sequential limit point. But no element of $G$ belongs to more than one of the collections $G_{i}$, each of which contains only a finite number of elements. Hence the sequence $h_{k(1), i(1)}, h_{k(2), i(2)}, h_{k(3), i(3)}, \cdots$ contains infinitely many distinct elements of $G$, and $P$ belongs to the limiting set of this sequence and thus to the limiting set of $G$. Thus we have demonstrated the sufficiency of the condition.

Suppose that there exists a space $L$ in which the limiting set of every collection of point sets is closed, but which is not a space $S$. Then there exists in this space a point set $M$ whose derived set has a limit point $P$ which does not belong to $M^{\prime}$. Let $W=P_{1}+P_{2}+P_{3}+\cdots$ be a sequence of distinct points belonging to $M^{\prime}$ and having $P$ as a sequential limit point. Then for each positive integer $i$ there exists a sequence $W_{i}=P_{1 i}+P_{2 i}+P_{3 i}+\cdots$ which has $P_{i}$ as a sequential limit point, is a sub-set of $M$, and does not contain $P$. Let $G$ be a collection whose elements are $P_{x y}$, where $x$ and $y$ are independent variables, each having as range the set of positive integers. Then $W$ is a sub-set of the limiting set of $G$, and so is $P$, since the limiting set is closed. It follows that $P$ is a limit point of $M$, contrary to its definition. Thus the condition is necessary.

Definition. A point $P$ is said to be a point of condensation $\dagger$ of a point set $M$ provided that every open set containing $P$ contains uncountably many points of $M$.

THEOREM 2. In order that it should be true for each infinite collection of point sets in a space $D$ that at most a countable number of its elements fail to

\footnotetext{
- For typographical reasons when $n_{i}$ and $n_{i j}$ occur as subscripts or superscripts they will be printed $n(i)$ and $n(i, j)$ respectively.

† Cf. Lindelöf, loc. cit., p. 184, and Fréchet I, p. 6.
} 
be sub-sets of its limiting set, it is necessary and sufficient that the space be separable.

We shall show first that the condition is sufficient. Let $K$ be the limiting set of a collection $G$ of point sets in a separable space $D$. Suppose that there exists an uncountable sub-collection $\bar{G}$ of the elements of $G$ such that no element of $\bar{G}$ is a sub-set of $K$. There must exist a positive number $y$ such that there exist uncountably many elements of $\bar{G}$ containing points whose distances* from $K$ are greater than $y$. Let $E$ be the set of all points belonging to elements of $\bar{G}$ and having distances from $K$ greater than $y$. If a point of $E$ is common to infinitely many elements of $\bar{G}$ it belongs to the limiting set of $G$. This is clearly impossible. Further, if $E$ contains at most a countable number of points, it follows from what we have just proved that at most a countable number of elements of $\bar{G}$ contain points in $E$; this involves a contradiction with our definition of $E$. Hence $E$ contains uncountably many points, and has a point of condensation. $\dagger$ Since no point of $E$ belongs to more than a finite number of elements of $\bar{G}$, this point of condensation belongs to the limiting set of $G$. Since its distance from $K$ is not less than $y$ we have a contradiction. Hence $\bar{G}$ does not exist, and the condition given in the statement of the theorem is sufficient.

We shall show next that the condition is necessary. Suppose that it is not, and that there exists a non-separable space $D$ in which every infinite collection of point sets has the property mentioned in the statement of the theorem. Then the space contains an uncountable point set $H$ having no limit point.f Let $G$ be a collection of elements $g$ such that each $g$ consists of a single point belonging to $H$ and each point of $H$ belongs to a single element of $G$. It follows that $H^{\prime}$ is the limiting set of $G$. From the fact that all except a countable number of the elements of $G$ are sub-sets of $H^{\prime}$ it follows that $H^{\prime}$ is non-vacuous. Thus, the supposition that the space is not separable has led to a contradiction.

Definition. A space is said to have the Cantor-Bendixson property provided that every closed point set in it is the sum of a perfect set and a countable set. The fact that this property holds in a euclidean space is, as

* If $P$ is a point and $N$ is a point set, by $d(P, N)=d(N, P)$, the distance from $P$. to $N$, is meant the lower bound of the set of values $[d(P, Q)]$, where $Q$ is a point of $N$.

$\dagger$ Cf. W. Gross, Zur Theorie der Mengen in denen ein Distanzbegriff definiert ist, Sitzungsberichte der Kaiserlichen Akademie der Wissenschaften, Mathematisch-Naturwissenschaftliche Klasse, vol. 123 (1914), IIa, pp. 805-806, and Fréchet I, p. 6.

‡ Cf. Gross, loc. cit., pp. 805-806. 
indicated by Lindelöf, loc. cit., the most important part of a theorem by Cantor* and Bendixson.

Theorem 3. In order that a space $D$ should have the Cantor-Bendixson property it is necessary and sufficient that the space be separable.

The sufficiency of the condition follows from the work of Fréchet $†$ and Gross †; the argument given by Fréchet is similar to one given for the euclidean case by Lindelöf (loc. cit., pp. 183, 187). The necessity of the condition follows easily from Gross's work. Suppose that there exists a space $D$ which has the Cantor-Bendixson property but is not separable. Then it contains an uncountable point set $M$ which has no limit point; $\ddagger$ then $M$ is closed. It follows that $M$ is the sum of a perfect set and a countable set. Since $M$ contains uncountably many points, this perfect set is non-vacuous. Every point of the perfect set is a limit point of this set and of $M$. Thus we get a contradiction.

THEOREM 4. In order that every infinite collection of point sets in a space $D$ should contain a countable sub-collection having the same limiting set as the collection itself it is necessary and sufficient that the space be separable.

It was pointed out by W. L. Ayres that the sufficiency of the condition for a bounded euclidean space is established as a consequence of the fact that such a space has the countably distributive property. The same conclusion holds for any space $D$ that has this property. Not all separable spaces $D$ have this property, however; cf. Theorem 9.

Let $G$ be a collection of point sets in a separable space $D$, and $K$ be its limiting set. Since the space is separable there exists a countable $\S$ sequence $P_{1}+P_{2}+P_{3}+\cdots$ every element of which is a point of $K$, and such that every point of $K$ is the sequential limit point of a subsequence of it. For each positive integer $n$ there exists an element $g_{n}$ of $G$ whose distance from $P_{n}$ is less than $1 / n$ and which is distinct from $g_{1}, g_{2}, g_{8}, \cdots, g_{n-1}$, the elements associated respectively with $P_{1}, P_{2}, P_{3}, \cdots, P_{n-1}$. Then $K$ is the limiting set of $g_{1}+g_{2}+g_{3}+\cdots$.

The necessity of the condition may be established as follows: Let $G$ be a collection of point sets in a space $D$ such that (1) each element of $G$ is a

* Cf. G. Cantor, Fondements d'une thérze generale des ensembies, Acta Mathematica, vol. 2 (1883), pp. 405, 409-414; and I. Bendixson: Quelques theorèmes de la theorie des ensembles de points, Acta Mathematica, vol. 2 (1883), pp. 415-427.

$\dagger$ Cf. Fréchet I, p. 19, and Gross, loc. cit., pp. 805-806, 815-816.

$\ddagger$ Cf. Gross, loc. cit., pp. 805-806.

§ Cf. Gross, loc. cit., pp. 805-806, and Fréchet I, p. 27. 
single point of the space and (2) for each point $x$ of the space there exists a countable sequence $g_{1 x}, g_{2 x}, g_{3 x}, \cdots$ of elements of $G$, each of which is the point $x$. Clearly the limiting set of $G$ is $M$, the set of all points in the space. By our hypothesis $G$ contains a countable sub-collection $G_{1}$ which has the same limiting set. It follows from our definition of a limiting set that if $y$ is a point of $M$ and is not the limit of an infinite sequence of distinct points belonging to $M_{1}$, the set of all points belonging to elements of $G_{1}$, it is the limit of a sequence $y, y, y, \cdots$ of points of $M_{1}$, each of which is the point $y$. Then $y$ belongs to $M_{1}$. Since $M_{1}$ is countable, $M$ is separable.

THEOREM 5. If, in a space $D$, the limiting set of a collection $G$ of point sets is separable, then every infinite sub-collection of $G$ contains a sub-collection that has a sequential limiting set.

The question as to the truth of this theorem for a bounded euclidean space was raised by R. L. Moore, and in June, 1925, was proved independently by him and by me; he pointed out that my method of proof bas an advantage over his in that it applies to abstract spaces. After submitting this paper for publication I learned that $\mathrm{T}$. Ważewski has the following theorem in his thesis:* In a compact space $D$ every infinite collection of point sets contains a convergent sub-collection. Also, since this paper was submitted for publication Zarankiewicz $\dagger$ and Hausdorff have published analogous results, Zarankiewicz showing that in a euclidean space and Hausdorff that in a separable space $D$ every infinite collection of point sets contains a sub-collection which has a sequential limiting set. The argument given by Hausdorff does not suffice to prove the theorem we have stated, since he uses the fact that the complement of the limiting set is separable; however, if the proof given by Zarankiewicz, which is similar to the one discovered by the author, is modified slightly, it suffices for our purposes.

In 1909 Zorettif proved the following theorem: If, in a plane, $G$ is a collection of continua (1) whose sum is bounded, (2) whose limit set is nonvacuous, and (3) whose limiting set is a continuum, then $G$ contains an inp. 24.

* Sur les courbes de Jordan ne renfermant aucune courbe simple fermée de Jordan, Krakow, 1923,

† Cf. Zarankiewicz, Sur les points de division dans les ensembles connexes, Fundamenta Mathematicae, vol. 9 (1927), pp. 127-129; and Hausdorff II, pp. 147-148.

$\ddagger$ Cf. Zoretti II.

$\$$ A continuum is a closed and connected point set. (Cf. Cantor, loc. cit., p. 406.) A point set is connected provided that it is not the sum of two mutually exclusive, non-vacuous point sets, neither of which contains a limit point of the other. Cf. N. J. Lennes, Curves in non-metrical analysis situs with an application in the calculus of variations, American Journal of Mathematics, vol. 33 (1911), p. 303, and Hausdorff I, p. 244. 
finite sub-collection which has a closed and connected sequential limiting set. The conditions (2) and (3) are unnecessary, for the existence of a sequence having property (2) follows from (1), and (3) follows from (2) by a theorem of Zoretti's, p. 116, loc. cit. In his process of selecting a sequence which has a sequential limiting set, Zoretti makes no use of the fact* that the elements of his collection are continua, and this process is adequate to prove that every infinite collection of point sets in a plane whose sum is bounded contains a convergent sub-collection.

Proof of Theorem 5. Let $K$ be a separable point set in a space $D$ and $G$ be a collection of point sets having $K$ as a limiting set. Then $K$ has a countable sub-set $H$ such that if $X$ is a point of $K$ it is either a point of $B$ or a limit point of $H$. For each point of $B$ consider all the spheres $\dagger$ having this point as a center and having radii whose lengths are rational numbers. Let $R_{1}, R_{2}, R_{3}, \cdots$ be the set of the interiors of these spheres. Now proceed as in the paper of Zarankiewicz (loc. cit., pp. 128-129).

THEOREM 6. In a space $L$ which contains a point set having the power of the continuum and having a vacuous derived set there exists an infinite collection of point sets which has no convergent or divergent sub-collection.

The following example shows that there exist spaces $D$ which satisfy the hypothesis of the theorem: Let $D_{1}$ be an aggregate of objects having the power of the continuum in which all the requirements of a space $D$ are satisfied, subject to the condition that the distance between two distinct elements of $D_{1}$ is unity.

Proof of Theorem 6. Consider a space $L$ in which there exists a point set $M$ having the power of the continuum, but having no limit point. Let $X$ be the set of all real numbers $x$ such that $0<x<1$. Then there exists a correspondence such that to each $x$ there corresponds exactly one point $P_{z}$ of $M$ and conversely. Let the number $x$ be expanded as an infinite decimal in the binary scale. Let $G$ be a collection of point sets, $g_{1}, g_{2}, g_{3}, \cdots$, where the set $g_{n}$ consists of all points of $M$ for which the corresponding elements in $X$, when given the binary expansion just mentioned, have as their $n$th digit unity.

Let $T=g_{n(1)}+g_{n(2)}+g_{n(8)}+\cdots$ be a sub-collection of $G$ such that $n_{1}<n_{2}<n_{3}<\cdots$. Let $z=\sum 2^{-n(2 i)}(i=1,2,3, \cdots)$, and $H=\sum g_{n(2 i)}(i=1$, $2,3, \cdots)$. Then $P_{s}$ is common to all the elements of $H$ and hence belongs to the limiting set of $\boldsymbol{H}$. Hence the limiting set of $T$ is non-vacuous, and $T$

* This fact is used in proving that the limiting set is connected.

$\dagger$ The terms sphere, interior and exterior of a sphere, etc. are defined precisely as in euclidean geometry. Cf. Fréchet I, p. 21, paragraph 34. A spherical region is the interior of a sphere. 
is not divergent. It follows from our definitions of $M$ and $G$ that a point of $M$ belongs to the limiting set of an infinite sub-collection of $G$ if and only if the point belongs to infinitely many elements of this sub-collection. Hence $P_{z}$ does not belong to the limiting set of $T-H$. Hence $T$ does not converge.

THEOREM 7. If the hypothesis of the continuum* is correct, then in order that every infinite collection of point sets in a space $D$ should contain a convergent or a divergent sub-collection, it is necessary and sufficient that the space be separable.

The theorem is a consequence of Gross, pp. 805-806, Fréchet I, p. 27, and Theorem 6, and Hausdorff II, pp. 147-148.

TheOREM 8. Every space $D$ which has the distributive property is locally compact.

If the theorem is not true, there exists a space $D$ having the distributive property, and containing a point $P$ near which it is not compact and a countably infinite set of points, $K$, which has no limit point and does not contain $P$. Let $R_{1}$ be a spherical region containing $P$ such that $\bar{R}_{1}$ contains no point of $K$. There exists in $R_{1}$ a countable point set $M_{1}$ having no limit point and not containing $P$; within $R_{1}$ there is a spherical region $R_{2}$ with its center at $P$, of diameter less than one half, and containing no point of $M_{1}$. This process may be continued; in general, let $R_{n}$ be a spherical region of diameter less than $1 / n$, which contains no point of the point sets $M_{1}, \dot{M}_{2}, M_{3}, \cdots$, $M_{n-1}$, has its center at $P$, and lies within the region $R_{n-1}$; let $M_{n}=P_{1 n}$ $+P_{2 n}+P_{3 n}+\cdots$ be a countable sequence of points which lies within $R_{n}$, does not contain $P$, and has no limit point. Let $K=P_{1}+P_{2}+P_{3}+\cdots$. For every pair of integers $i$ and $j$ let $g_{i j}=P_{i j}+P_{j}$. Then $P_{j}$ is the limiting set of $\sum g_{i j}(i=1,2,3, \cdots, \infty)$. From the fact that the space under consideration has the distributive property and that $K$ is closed, since it has no limit point, it follows that $K$ is the limiting set of a sub-collection $N$ of $\sum \sum g_{i j}(i$ and $j=1,2,3,4, \cdots, \infty)$. Then there exists an integer $m$ such that $R_{m}$ contains no points belonging to elements of $N$. It follows that $N$ is a sub-collection of $\sum \sum g_{i j}(j=1,2,3, \cdots, m-1 ; i=1,2,3, \cdots, \infty)$. There exists a spherical region $R$ which contains $P_{m+1}$ but contains no point of $K-P_{m+1}$ or of $\bar{R}_{1}$. But $P_{m+1}$ does not belong to any point sets in the collection $\sum \sum g_{i i}(j=1,2,3, \cdots, m-1 ; i=1 ; 2,3, \cdots, \infty)$. It follows

\footnotetext{
* The hypothesis of the continuum, as formulated by Cantor, is the following: No uncountable point set has a power less than that of the continuum. Sierpinski, Sur l'hypothese du contimu, Fundamenta Mathematicae, vol. 5 (1924), p. 177-187, gives a discussion of a number of interesting theorems whose truths follow from that of this hypothesis.
} 
that $P_{m+1}$ does not belong to the limiting set of $N$. Thus, the supposition that the space is not locally compact has led to a contradiction.

THEOREM 9. In order that a space D should have the countably distributive property it is necessary and sufficient that it be locally compact and separable.

First we shall show the sufficiency of the condition. Let $K$ be a closed point set in a separable, locally compact space $D$ and $G$ be a collection of point sets such that each point of $K$ belongs to a sub-set of $K$ which is the limiting set of some infinite sub-collection of $G$. Let $M$ be the limiting set of $G$ and $N=M-K$. The point set $K$ is separable* and hence there exists a countable sub-set $H=P_{1}+P_{2}+P_{3}+\cdots$ of $K$ such that $K \subset \bar{H}$. For each integer $i$ there exists a sub-collection $G_{i}=g_{1 i}+g_{2 i}+g_{3 i}+\cdots$ of $G$ such that the limit set of $G_{i}$ is a sub-set of $K$ and contains $P_{i}$.

From the fact that $K$ is closed and that the space is locally compact it follows that for each point $Q$ of $N$ there exists a spherical region $R_{Q}$ which is compact and such that $\bar{R}_{Q}$ contains no point of $K$. From the fact that the space is separable and hence has the Lindelöf property $\dagger$ it follows that there exists a countable sequence $R_{1}, R_{2}, R_{3}, \cdots$ of these regions covering $N$. If $i$ and $j$ are integers, the region $R_{j}$ contains points of at most a finite number of elements of $G_{i}$; for otherwise, since $\bar{R}_{j}$ is compact, it would contain a point of the limiting set of $G_{i}$, thus contradicting the fact that the limiting set of $G_{i}$ is a sub-set of $K$ and $\bar{R}_{j}$ contains no point of $K$. Hence, for every integer $i$ the sequence $G_{i}$ contains an infinite sub-sequence $F_{i}$ such that no element of $F_{i}$ contains a point in any of the regions $R_{1}, R_{2}, R_{3}, \cdots, R_{i}$. Let $F=\sum F_{i}$ $(i=1,2,3, \cdots, \infty)$. We shall show that $E$, the limiting set of $F$, is a sub-set of $K$. If it is not, it must contain a point $W$ of $N$, since $F$ is a sub-collection of $G$ and $E$ is then a sub-set of $M$. There exists an integer $m$ such that $W$ belongs to $R_{m}$. Let $n \geqq m$. Then it follows from the definition of $F_{n}$ that $F_{n}$ contains no point within $R_{m}$. Further, as we have pointed out above, if $1 \leqq p \leqq m$, at most a finite number of the elements of $G_{p}$, and hence of $F_{p}$, contain points within $R_{m}$. Hence $R_{m}$ contains points of at most a finite number of elements of $F$, and hence $W$ cannot belong to the limiting set of $F$.

We shall show next that $K$ is a sub-set of the limiting set of $F$. It follows from the definition of $F$ that $H$ is a sub-set of the limiting set of $F$. If $X$ is a point of $K-H$, it is a limit point of $H$. By Theorem $1, X$ is then a point of the limiting set of $F$. From $E \subset K$ and $K \supset E$ it follows that $K=E$.

Given a space $D$ which has the countably distributive property. If $G$

* Cf. Fréchet I, p. 27, and Gross, pp. 805-806.

† Cf. Gross, pp. 805, 806, 809. 
is a collection of point sets, it follows by Theorem 1 that its limiting set is closed. By our hypothesis $G$ can be replaced by a countable sub-collection having the same limiting set. By Theorem 4 the space is separable. By Theorem 8 the space is locally compact. Hence, the condition is necessary.

Definitions. If $M$ and $N$ are two point sets in a space $D$, by $l(M, N)$, the lower distance from $M$ to $N$, is meant the lower bound of the values $d(P, N)$, where $P$ is a point of $M$; and by $u(M, N)$, the upper distance from $M$ to $N$, is meant the upper bound of the values $d(P, N)$. If $G$ and $H$ are collections of point sets, we shall say that $H$ is upper semi-continuous with respect to $G$ provided that if $g$ is a fixed element of $G$ and $h$ is a variable element of $H$, then as $l(h, g)$ approaches zero so does $u(h, g)$. If the collection $G$ is upper semi-continuous with respect to itself, it is said to be an upper semi-continuous* collection.

TheOREM 10. If in a space $D, K$ is a closed point set and $G$ is a collection of point sets, then a sufficient condition that $K$ be the limiting set of $G$ is that $K$ is a sub-set of the limiting set of $G$ and (1) contains points in common with the limiting set of every infinite sub-collection of $G$ that has a non-vacuous limiting set; (2) $G$ is upper semi-continuous with respect to the point set $K$. If it be specified in addition that the space be compact, the condition mentioned above is both necessary and sufficient.

It is easy to construct examples showing that the condition mentioned in the theorem does not remain necessary in case the stipulation that the space be compact be removed. The theorem remains true if we replace (1) by $\left(1^{\prime}\right)$ following: ( $\left(1^{\prime}\right)$ If $e$ is a positive number, there exist at most a finite number of elements $g$ of $G$ such that $l(g, K)>e$.

Proof of Theorem 10. Let $B$ be the limiting set of $G$. If $B$ contains a point $x$ not belonging to $K$, there must exist an infinite sub-sequence $G_{x}=g_{1}+g_{2}+g_{3}+\cdots$ of $G$ such that every element of this sequence is at a distance from $x$ less than $\frac{1}{2} d(x, K)>0$, and for each positive integer $i$, $d\left(x, g_{i}\right)<1 / i$. By the hypothesis there exist (1) a positive number $d$ such that when $l(g, K)<d$ then $u(g, K)<\frac{1}{2} d(x, K)$, and (2) an infinite sub-sequence of elements of $G_{x}$, every element of this sub-sequence being at a lower distance from $K$ less than $d$. Thus the supposition that the point $x$ exists has led to a contradiction, and $K=H$.

* For definitions of lower distance, of upper distance, and of upper semi-continuous collections of point sets, cf. R. L. Moore, Concerning upper semi-continuous collections of continua which do not separate a given continuum, Proceedings of the National Academy of Sciences, vol. 10 (1924), p. 356, and Concerning: upper semi-continuous collections of continua, these Transactions, vol. 27 (1925), p. 416. 
We shall now show that if the space is compact the condition is necessary. Part (1) of the condition follows immediately. If part (2) does not follow, there must exist a positive number $\epsilon$ and a sub-sequence $H=H_{1}+H_{2}+H_{8}$ $+\cdots$ of elements of $G$ such that, for every $n, u\left(H_{n}, K\right)>\epsilon$, but $l\left(H_{n}, K\right)$ approaches zero as $n$ approaches infinity. Since the space is compact it follows that the limiting set of $H$ contains a point $x$ whose distance from $K$ is not less than $\epsilon$. But the result just stated involves a contradiction of our assumption that $K$ is the limiting set of $G$ and hence contains the limiting set of $H$.

Definitions. If $x$ and $y$ are a pair of points, a sequence of points $x_{1}(=x)$, $x_{2}, x_{3}, \cdots, x_{n}(=y)$ is said to be $a$ chain joining $x$ and $y$; a pair of consecutive points, $x_{j}$ and $x_{j+1}$, is said to be a link of this chain and $d\left(x_{j}, x_{j+1}\right)$ is the length of this link. If $g$ is a point set let $f(g)$ be the lower bound of the set of all positive numbers $h$ such that any pair of points in $g$ can be joined by a chain every point of which belongs to $g$ and every link of which has a length less than or equal to $h$. If $g$ is a bounded point set, $f(g)$ certainly exists; otherwise it need not. If $G$ is zusammenhängend* in the sense of Cantor, $f(g)=0$.

Let $M$ be a point set in a space $L$ and $P$ and $Q$ be points of $M$. Let $C_{P Q}=\sum \sum P_{i j}\left(j=1,2,3, \cdots, \infty ; i=1,2,3, \cdots, n_{j}\right)$ be a sub-set of $M$ such that for each positive integral value of $j, P_{1 j}=P, n_{i}$ is a positive integer, and $P_{n(i), i}=Q$. The points $P$ and $Q$ are said to be well chained in $M$ provided that there exists at least one $C_{P Q}$ such that if $j_{1}<j_{2}<j_{3}<\cdots$ and the sequence $\sum P_{m(j(k)), j(k)}(k=1,2,3, \cdots, \infty)$, of the set $C_{P Q}$ has a sequential limit point $T$, then $T$ is a sequential limit point also of every infinite subsequence of $\sum P_{m(j(k))+1, j(k)}(k=1,2,3, \cdots, \infty)$ and of $\sum P_{m(j(k))-1, j(k)}$ $(k=1,2,3, \cdots, \infty)$, where $1 \leqq m_{j(k)} \leqq n_{j(k)}$. If every pair $P$ and $Q$ of points of $M$ is well chained in $M, M$ is said to be well chained.

Theorem 11. If, in a locally compact space $D, G=[g]$ is a collection of point sets, $K$ is the limiting set of $G$, and $f(g)$ approaches zero as $l(g, K)$ approaches zero, then in order that $K$ be well chained it is necessary and sufficient that there exist a well chained sub-set $K_{1}$ of $K$ which contains points in common with every sub-set of $K$ which is the limiting set of a sub-collection of $G$.

Janiszewski considers an analogous question (1) for a bounded number space and (2) in an abstract space which may be characterized as a perfectly compact, connected space in which a distance is defined (cf. Janiszewski, p. 162, loc. cit.); he shows that in such spaces a sufficient condition that

- Cf. Cantor, loc. cit., p. 406, and Ueber unendliche, lineare Punktmannigfaltigkeiten, Mathematische Annalen, vol. 21 (1883), p. 576. 
the limiting set be connected is that the limit set exist. Our theorem is more general than his in that we give necessary and sufficient conditions and that our space is more general.

Schoenflies (loc. cit.) gives a less general case than Janiszewski; he assumes that the sequential limiting set exists. See also Zoretti I, pp. 8-9. and Zoretti II. In connection with Zoretti's work see our comments following Theorem 5. See also Vietoris, loc. cit., p. 186, and Hausdorff I, pp. 301302.

It might be pointed out that in a non-compact space $D$ the limiting set of a collection of connected point sets need not be connected, cf. Hausdorff I, p. 239, last four lines. As an example of a bounded space $D$ where the condition given in Theorem 11 is not sufficient consider the space $D_{3}$ given below. Note, however, that this space is not locally compact. Let $H_{x}$ be the straight line interval in the euclidean plane having as end points $(x, 0)$ and $(x, 1)$. Let $A$ be the point $(0,0)$ and $B$ the point $(0,1)$. Let the points of $D_{3}$ be the points in the euclidean sense of $A+B+\sum H_{1 / i}(i=1,2,3, \cdots, \infty)$, and distance and limit be defined as in ordinary space. Let $G$ be a collection of point sets whose elements are the $B$ 's in $\sum H_{1 / i}(i=1,2,3, \cdots, \infty)$. Then $G$ is a collection of connected point sets having as a sequential limiting set the sum of $A$ and $B$. The set $A+B$ is neither connected nor well chained.

Proof of Theorem 11. Obviously the condition is necessary; we shall prove its sufficiency.

First we shall show that if $M$ is a connected point set it is "zusammenhängend." For, if it is not, there exists a positive number $d$ and a pair of points $x$ and $y$ of $M$ which cannot be joined by a finite chain of points belonging to $M$, each link in the chain having a length less than $d$. Let $N$ be the set of all points of $M$ which can be joined to $x$ by a chain such as that just mentioned. Then both $N$ and $M-N$ are non-vacuous and, since $M$ is connected, there exists a point $z$ which belongs to one of the point sets $N$ and $M-N$ and is a limit point of the other. Let $E$ be a spherical region with center at $z$ and having a radius of length $d / 3$. It follows that $E$ contains points of both $N$ and $M-N$. Clearly this involves a contradiction. Evidently $M$ is well chained.

We shall show next that if $M$ and $N$ are two non-compact, connected point sets their sum is well chained. Let $m_{1}, m_{2}, m_{3}, \cdots$ and $n_{1}, n_{2}, n_{3}, \cdots$ be two infinite sequences of points belonging to $M$ and $N$ respectively, and neither of which has a limit point. It follows from the preceding paragraph that for each positive integer $i$ there exists a pair of chains of points $m_{1 i}\left(=m_{1}\right)$, $m_{2 i}, m_{3 i}, \cdots, m_{p(i), i}\left(=m_{i}\right)$ and $n_{1 i}\left(=n_{1}\right), n_{2 i}, n_{3 i}, \cdots, n_{q(i), i}\left(=n_{i}\right)$ be- 
longing to $\dot{M}$ and to $N$ respectively such that each link in each of these chains has a length less than $1 / i$. Let $C_{i}$ be the chain $m_{1 i}, m_{2 i}, m_{3 i}, \cdots$, $m_{p(i), i}, n_{q(i), i}, \cdots, n_{2 i}, n_{1 i}$. It follows from the definitions of $C_{1}, C_{2}, C_{3}, \cdots$ that $m_{1}$ and $n_{1}$ are well chained in $M$, and since they may be selected at pleasure it follows that $M+N$ is well chained.

Hence, to prove that $K$ is well chained it suffices to prove that either (1) $K$ is connected or (2) every maximal* connected sub-set of $K$ is non-compact. Suppose that neither of these conditions is realized. Then $K$ has a compact maximal connected sub-set $T$ which does not contain all of $K$. About each point of $T$ there exists a spherical region such that the sum of its points and limit points is compact. The point set $T$ can be covered by a finite subcollection of this collection of regions. Let $H$ be the sum of the points belonging to this finite set of regions and $B$ be the boundary $\ddagger$ of $B$. Evidently $B$ contains no point of $T$. Since $\bar{H}$ is the sum of a finite number of compact point sets, it is compact. Let $G_{2}$ be a sub-collection of $G$ having a limit set containing a point $P$ of $T$. Suppose that $K_{2}$, the limiting set of $G_{2}$, contains points without $\bar{H}$; let $Q$ be such a point. Let $d$ be a positive number which is less than each of the numbers $d(P, h)$ and $d(Q, H)$. It follows from the hypothesis of the theorem and the definition of $G_{2}$, that $G_{2}$ contains a subsequence $g_{1}, g_{2}, g_{3}, \cdots$ such that for each positive integer $n, f\left(g_{n}\right), d\left(P, g_{n}\right)$, and $d\left(Q, g_{n}\right)$ are each smaller than the smaller of $d / 2$ and $1 / n$. For each $n$ there exists a finite chain $F_{n}=x_{1 n}, x_{2 n}, x_{3 n}, \cdots, x_{i(n) n}$ of points belonging to $g_{n}$ such that (1) all except the last point in this chain belong to $\bar{H}$ but the last does not, (2) the distance of $x_{1 n}$ from $P$ is less than $1 / n$, and (3) each link has a length less than $1 / n$. The limiting set $F$ of $\sum F_{n}(n=1,2,3, \cdots, \infty)$ is closed and connected, $\|$ contains $P$, and is a sub-set of $T$, which is, by definition, a maximal connected sub-set of $K$. The point set $\sum x_{i(n)-1, n}(n=1$, $2,3, \cdots, \infty)$ is compact and has the same limit points as $\sum x_{i(n), n}(n=1$, $2,3, \cdots, \infty)$. These points are common to $K_{2}, T$, and $B$. But $B$ and $T$ have no points in common, and hence $K_{2} \subset \bar{H}$.

Further $K_{2} \subset T$. For, if $e$ is greater than zero, we can evidently cover $T$ with a set of spherical regions each of which is compact and has a diameter less than $e$. In terms of these regions we can then define a point set $\bar{H}_{\bullet}$ which has properties similar to those of $\bar{H}$, and which contains no point whose

* A maximal sub-set, having a property $P$, of a point set $M$, is a sub-set $N$ of $M$ which has the property $P$ but is not a proper sub-set of any sub-set of $M$ which has this property.

$\dagger$ Cf. Gross, p. 810, and Fréchet I, pp. 22 and 26.

$\mp$ That is, $B$ is the set of all limit points of $H$ that do not belong to $H$.

$\S$ Let $h$ be the complement of $\bar{H}$.

H Cf. Janiszewski, loc. cit., p. 98, lines 1-12. 
distance from $T$ is greater than $e$. As above, $K_{2} \subset \bar{H}_{e}$. Then $K_{2} \subset T=\prod \bar{H}_{1 / i}$ $(i=1,2,3, \cdots, \infty)$.

It follows from the hypothesis of the theorem that $K_{2}$, and hence $T$, contains points of $K_{1}$. By an argument analogous to that used in showing that $K_{2} \subset T$ we can show that $K_{1} \subset T$.

If there exists a point $Y$ of $K-T$, there exists an infinite sub-collection $G_{Y}$ of $G$ having a limit set containing $Y$. It follows from the hypothesis of the theorem that $G_{Y}$ contains an infinite sub-collection having a limit set containing a point of $K_{1}$. We have shown above that the limiting set of such a collection is a sub-set of $T$. Hence the point $Y$ does not exist.

From $T \subset K$ and $K \subset T$ it follows that $K=T$. But this contradicts our supposition that $K$ is not well chained. Thus we have established the truth of our theorem.

THEOREM 12. In order that a compact point set in a locally compact space $D$ should be the closed and connected limiting set of a collection of well chained point sets, it is necessary and sufficient that it (1) be a maximal well chained sub-set of the limiting set of this collection and (2) contain points in common with the limiting set of every infinite sub-collection of the given collection which has a non-vacuous limiting set.

It is a consequence of the definition of well-chainedness that if $P$ is a limit point of a point set $M$ then $M+P$ is well chained. Let $K$ be the limiting set of a collection of point sets and $K_{1}$ be a maximal well chained sub-set of $K$. By Theorem 1, $K$ is closed. Since $K_{1}$ is a maximal well chained sub-set of $K$, it is closed. The argument used in proving Theorem 11 shows that the condition is sufficient. Its necessity is obvious.

If we leave off the condition that the space $D$ under consideration be locally compact, the condition given in Theorem 12 is not sufficient. Consider, for example, the space $D_{3}$ mentioned in the discussion following the statement of Theorem 11. The point $A$ satisfies the condition but is not the limiting set of any sub-collection of $\sum H_{1 / i}(i=1,2,3, \cdots, \infty)$. The point just raised applies also to each of the following two theorems.

Theorem 13. If $G=[g]$ is a collection of point sets in a separable, locally compact space $D, K$ is the limiting set of $G, f(g) \rightarrow 0$ as $l(g, K) \rightarrow 0$, and $K_{1}$ is a maximal well chained sub-set of $K$, then there exists a countable subcollection of $G$ whose limiting set is $K_{1}$.

Let $P$ be a point of $K_{1}$. There exists a sub-collection $G_{P}$ of $G$ whose limit set exists and contains $P$. Suppose that there exists a point $Q$ of $K_{P}$, the limiting set of $G_{P}$, such that $Q$ is not a point of $K_{1}$. By Theorem 11, $P$ and 
$Q$ are well chained in $K_{P}$. But then $K_{1}$ is not a maximal well chained sub-set of $K$. Thus, the supposition that $K_{P}$ is not a sub-set of $K_{1}$ leads to a contradiction. The truth of the theorem follows by Theorem 9.

THEOREM 14. If $G$ is a collection of connected point sets in a compact space $D$ and $K_{1}$ is a maximal connected sub-set of the limiting set of $G$, there exists a countable sub-collection of $G$ whose limiting set is $K_{1}$.

Definitron. A collection $G$ of point sets is said to be a separable collection provided that it contains a countable sub-collection $H$ such that if $g$ is an element of $G$ it is either an element of $H$ or the limiting set of some infinite sub-collection of $H$.

TheOREM 15. If $G$ is a collection of closed point sets in a separable space $D$, a sufficient condition that $G$ be a separable collection is that it contain a subcollection $B$ such that (1) $B$ has the same limiting set as $G$, and (2) is upper semicontinuous with respect to $G$. In particular, if the collection $G$ just mentioned is upper semi-continuous, it is a separable collection.

The condition mentioned in the theorem is not necessary. Consider, for instance, the collection $G$ whose elements are the graphs in the euclidean plane of $x=0$ and of $y=n x$, where $n=1,2,3, \cdots$.

Let $K$ be the common limiting set of $G$ and $H$. By Theorem 2, $G$ is the sum of a pair of sub-collections $G_{1}$ and $G_{2}$, and $H$ of a pair of sub-collections $G_{3}$ and $G_{4}$ such that $G_{1}$ and $G_{3}$ contain at most a countable number of elements and every element of $G_{2}$ and of $G_{4}$ respectively is a sub-set of $K$. Since the space is separable, $K$ is separable and contains a countable sub-set $T=P_{1}$ $+P_{2}+P_{3}+\cdots$ such that every point of it either belongs to $T$ or is a limit point of $T$. For each positive integer $n$ there exists a countable sub-collection $H_{n}$ of $H$ having a limit set containing $P_{n}$. Let $G_{5}=\sum H_{n}(n=1,2,3, \cdots$, $\infty)$. If $g$ is an element of $G_{2}$, it contains a countable sub-set $T_{\theta}=Q_{1 \theta}+Q_{2}$, $+Q_{30}+\cdots$ such that every point of $g$ either belongs to $T_{v}$ or is a limit poin $t$ of it. For every positive integral $i$ the point $Q_{i \sigma}$ is a point or a limit point of a sub-sequence of $T$; it can easily be shown that $Q_{i o}$ belongs to the limit set of a sequence $M_{i \theta}$ belonging to $G_{5}$ such that no element of $M_{i o}$ is at a lower distance from $Q_{i o}$ greater than $1 / i$. By the note following Theorem $10, g$ is the limiting set of $\sum M_{i o}(i=1,2,3, \cdots, \infty)$. Hence $G_{1}+G_{b}$ is a subcollection of $G$ of the type required to make $G$ a separable collection.

THEOREM 16. The aggregate of all closed point sets in a space $L$ may itself be regarded as a space $L$ provided that "the limit of a sequence of elements" in this hyperspace be defined as the minimal closed set in the given space $L$ containing the non-vacuous sequential limiting set of the given sequence. 
If $M$ is a closed point set in a space $L$, the sequential limiting set of the sequence $M, M, M, M, \cdots$, all of whose elements are the point set $M$, is clearly $M$. Thus, the third condition in the definition of a space $L$ is satisfied in our hyperspace. If $N$ is a point set in a space $L$, there exists* a point set $K$ which is the minimal closed point set in that space containing $N$. From the results just indicated and the definition of a sequential limiting set it follows that our hyperspace satisfies the other requirements of a space $L$.

TheOREM 17. The aggregate of all closed point sets in a space $S$ is a space $L$ provided that "the limit of a sequence" is defined as the non-vacuous sequential limiting set of the sequence in the given space $S$.

The theorem follows from Theorems 1 and 16.

- Cf. Hausdorff II, p. 230.

UNIVERSITY OF TEXAS, Austin, Texas 